\title{
Brézis-Wainger Inequality on Riemannian Manifolds
}

\author{
Przemysław Górka \\ Department of Mathematics and Information Sciences, Warsaw University of Technology, \\ Pl. Politechniki 1, 00661 Warsaw, Poland \\ Correspondence should be addressed to Przemysław Górka, pgorka@mini.pw.edu.pl \\ Received 10 November 2007; Accepted 29 April 2008 \\ Recommended by Shusen Ding \\ The Brézis-Wainger inequality on a compact Riemannian manifold without boundary is shown. For \\ this purpose, the Moser-Trudinger inequality and the Sobolev embedding theorem are applied. \\ Copyright (C) 2008 Przemysław Górka. This is an open access article distributed under the Creative \\ Commons Attribution License, which permits unrestricted use, distribution, and reproduction in \\ any medium, provided the original work is properly cited.
}

\section{Introduction}

There is no doubt that the Brézis-Wainger inequality (see [1]) is a very useful tool in the examination of partial differential equations. Namely, a lot of estimates to a solution of PDE are obtained with the help of the Brézis-Wainger inequality. Especially, the inequality is often applied in the theory of wave maps.

In this paper, we extend the Brézis-Wainger result onto a compact Riemannian manifold. We show the following theorem.

Theorem 1.1. Let $(\mathcal{M}, g)$ be an $n$-dimensional compact Riemannian manifold, and $u \in H^{k, p}(\mathcal{M})$, $\int_{\mathcal{M}} u d V_{g}=0$, for $n>k>n / p$, where $k$ is a positive integer and $p \geq 1$ is a real number. Then, $u \in L^{\infty}(\mathcal{M})$ and

$$
\|u\|_{L^{\infty}(\mathcal{M})} \leq\|u\|_{H^{k, n / k}(\mathcal{K})}\left(C+\log \frac{\|u\|_{H^{k, p}(\mathcal{M})}}{\|u\|_{H^{k, n / k}(\mathcal{M})}}\right)
$$

where $C=C(k, \mathcal{M})$ is a positive constant.

The proof relies on the application of a Moser-Trudinger inequality (see Theorem 2.2) and the Sobolev embedding theorem (see Theorem 2.1). Moreover, we will use the integral representation of a smooth function via the Green function (see [2]). 


\section{Preliminaries}

In order to make this paper more readable, we recall some definitions and facts from the theory of Sobolev spaces on Riemannian manifolds. In particular, we present useful inequalities and embeddings.

Let $(\mathcal{M}, g)$ be a smooth, compact Riemannian manifold without boundary. We will denote by $C^{\infty}(\mathcal{M})$ a space of smooth real functions. For $\phi \in C^{\infty}(\mathcal{M})$ and integer $m$, we denote by $\nabla^{m} \phi$ the $m$ th covariant derivative of $\phi$. Next, for $\phi \in C^{\infty}(\mathcal{M})$ and for a fixed integer $m$ and a real $p \geq 1$, we set

$$
\|\phi\|_{H^{m, p}(\mathcal{M})}=\sum_{i=0}^{m}\left(\int_{\mathcal{M}}\left|\nabla^{i} \phi\right|^{p} d V_{g}\right)^{1 / p}
$$

where by $V_{g}$ we have denoted the Riemannian measure on the manifold $(\mathcal{M}, g)$.

We define the Sobolev space $H^{m, p}(\mathcal{M})$ as a completion of $C^{\infty}(\mathcal{M})$ with respect to $\|\cdot\|_{H^{m, p}(\mathcal{M})}$.

We close this section stating the following results, which will be used in the proof of the main result.

Theorem 2.1 (Sobolev embedding theorem $[3,4])$. Let $(\mathcal{M}, g)$ be a smooth, compact Riemannian $n$ manifold. Then, for any real numbers $1 \leq p<q$ and any integers $0 \leq m<k$, if $1 / q=1 / p-(k-m) / n$, then $H^{k, p}(\mathcal{M}) \hookrightarrow H^{m, q}(\mathcal{M})$. Moreover, there exists a constant $C$ such that for all $u \in H^{k, p}(\mathcal{M})$, the following inequality holds:

$$
\|u\|_{H^{m, q}(\mathcal{M})} \leq C\|u\|_{H^{k, p}(\mathcal{M})}
$$

Theorem 2.2 (Moser-Trudinger inequality [5]). Let $(\mathcal{M}, g)$ be a smooth, compact Riemannian $n$ manifold and $k$ a positive integer, strictly smaller than $n$. There exist a constant $C=C(k, \mathcal{M})$ and $\lambda(k, n)$ such that for all $u \in C^{n}(\mathcal{M})$ with $\int_{\mathcal{M}} u d V_{g}=0$ and $\int_{\mathcal{M}}\left|\nabla^{k} u\right|^{n / k} d V_{g} \leq 1$, the following inequality holds:

$$
\int_{\mathcal{M}} e^{\lambda(k, n)\left(u /\|u\|_{H^{k, n / k}(\mathcal{M})}\right)^{n /(n-k)}} d V_{g} \leq C .
$$

Let us stress that this inequality is a generalization of the Moser and Trudinger result (see [6-8]).

\section{Proof of the main result}

In this section, we will prove the main result, that is, Theorem 1.1.

Proof. Let us notice that by the assumptions we have an embedding $H^{k, p}(\mathcal{M}) \hookrightarrow H^{k, n / k}(\mathcal{M})$. First of all, we show the following lemma.

Lemma 3.1. If $u \in C^{\infty}(\mathcal{M})$, $\int_{\mathcal{M}} u d V_{g}=0, \int_{\mathcal{M}}\left|\nabla^{k} u\right|^{n / k} d V_{g} \leq 1$, and $d \in[1, \infty)$, then $e^{u} \in L^{d}(\mathcal{M})$. Moreover, the following estimate holds:

$$
\left\|e^{u}\right\|_{L^{d}(\mathcal{M})} \leq C\left(\|u\|_{H^{k, n / k}(\mathcal{M})}, d, \mathcal{M}\right) .
$$


Proof. Let us put

$$
\begin{aligned}
& a=\left(\lambda(k, n) \frac{n}{n-k}\right)^{(n-k) / n} \frac{u}{\|u\|_{H^{k, n / k}(\mathcal{M})}}, \\
& b=d\left(\lambda(k, n) \frac{n}{n-k}\right)^{n /(n-k)}\|u\|_{H^{k, n / k}(\mathcal{N})}
\end{aligned}
$$

in Young's inequality

$$
a b \leq \frac{a^{p}}{p}+\frac{b^{q}}{q}
$$

where $1 / p+1 / q=1$. Then, we obtain an estimate

$$
u d \leq \lambda(k, n)\left(\frac{u}{\|u\|_{H^{k, n / k}(\mathcal{M})}}\right)^{n /(n-k)}+\frac{k}{n} d^{n / k}\left(\lambda(k, n) \frac{n}{n-k}\right)^{(n-k) / n}\|u\|_{H^{k, n / k}(\mathcal{M})^{\prime}}^{n / k}
$$

where $\lambda(k, n)$ is a constant from the Moser-Trudinger inequality (see Theorem 2.2).

Next, we can estimate

$$
\int_{\mathcal{M}} e^{u d} d V_{g} \leq C\left(d,\|u\|_{H^{k, n / k}(\mathcal{M})}\right) \int_{\mathcal{M}} e^{\lambda(k, n)\left(u /\|u\|_{H^{k, n / k}(\mathcal{M})}\right)^{n /(n-k)}} d V_{g}
$$

Subsequently, we can apply the Moser-Trudinger inequality to the right-hand side of the above inequality:

$$
\int_{\mathcal{M}} e^{\lambda(k, n)\left(u /\|u\|_{H^{k, n / k}(\mathcal{M})}\right)^{n /(n-k)}} d V_{g} \leq c
$$

From this, the proof of Lemma 3.1 follows.

Now, we can go back to the proof of Theorem 1.1. First, we prove the theorem for $u \in$ $C^{\infty}(\mathcal{M})$ such that $\|u\|_{H^{k, n / k}(\mathcal{M})}=1$ and $\int_{\mathcal{M}} u d V_{g}=0$. Replacing $u$ by $-u$ if necessary, we may suppose that

$$
\|u\|_{L^{\infty}(\mathcal{M})}=u\left(x_{0}\right)
$$

for $x_{0} \in \mathcal{M}$.

Let us recall (see [2]) that for $(\mathcal{M}, g)$, a compact Riemannian $n$-manifold, there exists a Green function $\mathrm{G}: \mathcal{M} \times \mathcal{M} \rightarrow \mathbb{R}$ such that

(1) for any $\phi \in C^{\infty}(\mathcal{M})$ and any $z \in \mathcal{M}$,

$$
\phi(z)=\frac{1}{\operatorname{Vol}(\mathcal{M})} \int_{\mathcal{M}} \phi d V_{g}+\int_{\mathcal{M}} \mathrm{G}(z, x) \Delta_{g} \phi(x) d V_{g}
$$

where $\operatorname{Vol}(\mathcal{M})$ is a Riemannian volume of the manifold $(\mathcal{M}, g)$, and $\Delta_{g}$ is the LaplaceBeltrami operator on a manifold; 
(2) there exists a constant $C$ such that for any $(x, y) \in \mathcal{M} \times \mathcal{M} \backslash \Delta$,

$$
\left|\nabla_{x} \mathrm{G}(x, y)\right| \leq \frac{C}{\left(d_{g}(x, y)\right)^{n-1}}
$$

where $\Delta$ is a diagonal:

$$
\Delta=\{(x, y) \in \mathcal{M} \times \mathcal{M}: x=y\}
$$

and $d_{g}(x, y)$ is a Riemannian distance from $x$ to $y$.

Let us define the map $\psi=e^{u}-1$. Next, we apply the first property of the Green function to the map $\psi$ and to the point $x_{0}$. Namely,

$$
\begin{aligned}
0 & \leq \psi\left(x_{0}\right) \\
& =\frac{1}{\operatorname{Vol}(\mathcal{M})} \int_{\mathcal{M}}\left(e^{u}-1\right) d V_{g}+\int_{\mathcal{M}} \mathrm{G}\left(x_{0}, x\right) \Delta_{g} \psi(x) d V_{g} \\
& \leq 1+\frac{1}{\operatorname{Vol}(\mathcal{M})}\left\|e^{u}\right\|_{L^{1}(\mathcal{M})}+\int_{\mathcal{M}}\left|\nabla_{x} \mathrm{G}\left(x_{0}, x\right) \| \nabla_{x} \psi(x)\right| d V_{g} .
\end{aligned}
$$

Subsequently, by the second property of the Green function we can estimate $\psi\left(x_{0}\right)$ as follows:

$$
\psi\left(x_{0}\right) \leq 1+\frac{1}{\operatorname{Vol}(\mathcal{M})}\left\|e^{u}\right\|_{L^{1}(\mathcal{M})}+\int_{\mathcal{M}} \frac{C}{\left(d_{g}\left(x_{0}, x\right)\right)^{n-1}}|\nabla u| e^{u} d V_{g}
$$

Now, we will try to estimate the last term in the inequality (3.12). Let us notice that if $u \in$ $H^{k, p}(\mathcal{M})$, then $\nabla u \in H^{k-1, p}(\mathcal{M})$. Next, by the Sobolev theorem (see Theorem 2.1),

$$
H^{k-1, p}(\mathcal{M}) \hookrightarrow L^{q}(\mathcal{M}), \quad \text { for } \frac{1}{q}=\frac{1}{p}-\frac{k-1}{n}
$$

and we have that $\nabla u \in L^{q}(\mathcal{M})$.

Using elementary calculations, one can easily show the lemma.

Lemma 3.2. There exist $r<n /(n-1)$ and a finite $d$ such that the following equality holds:

$$
\frac{1}{r}+\frac{1}{q}+\frac{1}{d}=1
$$

where $q$ is the exponent from the Sobolev theorem. follows:

By Hölder's inequality with exponents $r, q, d$, we can estimate the inequality (3.12) as

$$
\psi\left(x_{0}\right) \leq 1+\frac{1}{\operatorname{Vol}(\mathcal{M})}\left\|e^{u}\right\|_{L^{1}(\mathcal{M})}+\tilde{C}\|\nabla u\|_{L^{q}(\mathcal{M})}\left\|e^{u}\right\|_{L^{d}(\mathcal{M})^{\prime}}
$$


where $\tilde{C}=\sup _{x_{0} \in \mathcal{M}}\left\|C /\left(d_{g}\left(x_{0}, \cdot\right)\right)^{n-1}\right\|_{L^{r}(\mathcal{M})}$ and $r$ is the exponent from Lemma 3.2. Finally, by Lemma 3.1 and the Sobolev theorem, we obtain

$$
\psi\left(x_{0}\right) \leq C\left(1+\|u\|_{H^{k, p}(\mathscr{M})}\right) .
$$

Let us stress that since $\|u\|_{H^{k, n / k}(\mathcal{M})}=1$, the constant $C$ does not depend on $u$. We can rewrite the inequality (3.16) as follows:

$$
e^{u\left(x_{0}\right)} \leq C\left(1+\|u\|_{H^{k, p}(\mathcal{M})}\right) .
$$

Hence,

$$
\|u\|_{L^{\infty}(\mathcal{M})}=u\left(x_{0}\right) \leq \log \left(C\left(1+\|u\|_{H^{k, p}(\mathcal{M})}\right)\right) .
$$

Taking into account $1=\|u\|_{H^{k, n / k}(\mathcal{M})} \leq c\|u\|_{H^{k, p}(\mathscr{M})}$, we obtain

$$
\|u\|_{L^{\infty}(\mathcal{M})} \leq C+\log \|u\|_{H^{k, p}(\mathscr{M})} .
$$

This finishes the proof of the inequality in the case $u \in C^{\infty}(\mathcal{M})$ such that $\|u\|_{H^{k, n / k}(\mathcal{M})}=1$ and $\int_{\mathscr{M}} u d V_{g}=0$. Subsequently, one can easily obtain the inequality for $u \in C^{\infty}(\mathcal{M})$ such that $\int_{\mathscr{M}} u d V_{g}=0$.

Now, we prove the theorem for an arbitrary $u \in H^{k, p}(\mathcal{M})$ such that $\int_{\mathcal{M}} u d V_{g}=0$. We apply the density argument. Namely, for any $\varepsilon>0$, there exists $\tilde{u}_{\varepsilon} \in C^{\infty}(\mathcal{M})$ such that

$$
\left\|\tilde{u}_{\varepsilon}-u\right\|_{H^{k, p}(\mathcal{M})} \leq \varepsilon .
$$

Next, we define

$$
u_{\varepsilon}=\tilde{u}_{\varepsilon}-\frac{1}{\operatorname{Vol}(\mathcal{M})} \int_{\mathcal{M}} \tilde{u}_{\varepsilon} d V_{g}
$$

Such $u_{\varepsilon}$ has zero-mean value. Moreover, the following inequality holds:

$$
\left\|u_{\varepsilon}-u\right\|_{H^{k, p}(\mathcal{M})} \leq 2 \varepsilon
$$

Indeed,

$$
\begin{aligned}
\left\|u_{\varepsilon}-u\right\|_{H^{k, p}(\mathcal{M})} & \leq\left\|\tilde{u}_{\varepsilon}-u\right\|_{H^{k, p}(\mathcal{M})}+\left\|\frac{1}{\operatorname{Vol}(\mathcal{M})} \int_{\mathcal{M}}\left(\tilde{u}_{\varepsilon}-u\right) d V_{g}\right\|_{H^{k, p}(\mathcal{M})} \\
& \leq \varepsilon+\frac{1}{\operatorname{Vol}(\mathcal{M})} \int_{\mathcal{M}}\left|\tilde{u}_{\varepsilon}-u\right| d V_{g}\|1\|_{H^{k, p}(\mathcal{M})} \\
& \leq \varepsilon+\left(\int_{\mathcal{M}}\left|\tilde{u}_{\varepsilon}-u\right|^{p} d V_{g}\right)^{1 / p} \\
& \leq 2 \varepsilon .
\end{aligned}
$$

Hence,

$$
\|u\|_{L^{\infty}(\mathcal{M})} \leq\left\|u-u_{\varepsilon}\right\|_{L^{\infty}(\mathcal{M})}+\left\|u_{\varepsilon}\right\|_{H^{k, n / k}(\mathcal{M})}\left(C+\log \frac{\left\|u_{\varepsilon}\right\|_{H^{k, p}(\mathcal{M})}}{\left\|u_{\varepsilon}\right\|_{H^{k, n / k}(\mathcal{M})}}\right) .
$$

Finally, we take $\varepsilon \rightarrow 0$. This completes the proof. 


\section{Acknowledgments}

The author wishes to thank Professor Yuxiang Li for pointing out the paper [5]. Moreover, the author thanks the referees for comments and invaluable suggestions.

\section{References}

[1] H. Brézis and S. Wainger, "A note on limiting cases of Sobolev embeddings and convolution inequalities," Communications in Partial Differential Equations, vol. 5, no. 7, pp. 773-789, 1980.

[2] T. Aubin, Nonlinear Analysis on Manifolds. Monge-Ampère Equations, vol. 252 of Grundlehren der Mathematischen Wissenschaften, Springer, New York, NY, USA, 1982.

[3] E. Hebey, Sobolev Spaces on Riemannian Manifolds, vol. 1635 of Lecture Notes in Mathematics, Springer, Berlin, Germany, 1996.

[4] E. Hebey, Nonlinear Analysis on Manifolds: Sobolev Spaces and Inequalities, vol. 5 of Courant Lecture Notes in Mathematics, American Mathematical Society, Providence, RI, USA, 2000.

[5] L. Fontana, "Sharp borderline Sobolev inequalities on compact Riemannian manifolds," Commentarii Mathematici Helvetici, vol. 68, no. 1, pp. 415-454, 1993.

[6] D. R. Adams, "A sharp inequality of J. Moser for higher order derivatives," The Annals of Mathematics, vol. 128 , no. 2, pp. 385-398, 1988.

[7] J. Moser, "A sharp form of an inequality by N. Trudinger," Indiana University Mathematics Journal, vol. 20, pp. 1077-1092, 1971.

[8] N. S. Trudinger, "On imbeddings into Orlicz spaces and some applications," Journal of Mathematics and Mechanics, vol. 17, pp. 473-483, 1967. 